\title{
Gender Difference is Associated with Short-
Outcomes in Non-Surgically Managed Acute Aortic Dissection Patients with Hypertension: A Retrospective Cohort Study
}

This article was published in the following Dove Press journal:

Risk Management and Healthcare Policy

\author{
Yang Zhou ${ }^{1,2, *}$ \\ Wen Peng ${ }^{1,2, *}$ \\ Guifang Yang ${ }^{1,2}$ \\ Xiaogao Pan ${ }^{1,2}$ \\ Ning Ding ${ }^{1,2}$ \\ Hongliang Zhang ${ }^{1,2}$ \\ Zhenyu Peng ${ }^{1,2}$ \\ Dongshan Zhang ${ }^{1,2}$ \\ Sijie $\mathrm{Wu}^{3}$ \\ Xiangping Chai ${ }^{1,2}$ \\ 'Department of Emergency Medicine, \\ The Second Xiangya Hospital, Central \\ South University, Changsha, People's \\ Republic of China; ${ }^{2}$ Emergency Medicine \\ and Difficult Diseases Institute, Central \\ South University, Changsha, People's \\ Republic of China; ${ }^{3}$ Department of \\ Cardiovascular Surgery, The Second \\ Xiangya Hospital, Central South \\ University, Changsha, People's Republic
} of China

*These authors contributed equally to this work
Correspondence: Sijie Wu; Xiangping Chai The Second Xiangya Hospital of Central South University, 139 Renmin Road, Changsha, Hunan Province 4100II, People's Republic of China

Email wusijie0605@sina.com;

chaixiangping@csu.edu.cn
Background: The management of acute aortic dissection (AAD) has improved; however, the outcomes related to different gender with short-term outcomes in non-surgically managed AAD with hypertension are still limited. Our objective was to explore gender-differences in association with short-term outcomes of patients comorbid with hypertension in non-surgically managed AAD. Methods: This is an observational retrospective single-center cohort. We analyzed the data from the Second Xiangya Hospital of Central South University (2014-2018). The data on demographics, clinical presentation, chronic comorbidities, laboratory testing, imaging studies, and treatment were analyzed for all patients. Univariate and multiple analyses were used to test gender-difference associated with short-term outcomes of patients with hypertension in non-surgically managed AAD.

Results: In total, 288 patients were enrolled in this study, of whom 238 (82.63\%) were male and $50(17.37 \%)$ were females. About $74 \%$ of female patients were dead in-hospital, which was more than male patients $(56.3 \%)$. Female patients with diabetes mellitus were more than male patients ( $14 \%$ vs $2.94 \%$ ), while male patients with smoking were significant higher than female patients (36.55\% vs $8 \%$ ). In the full model (model 3), after adjusting for confounding variables, the female AAD patients were more likely to have worse short-term outcomes ( $\mathrm{OR}=3.60,95 \% \mathrm{CI}=1.41$ to 9.60$)$.

Conclusion: Female patients were more likely to have worse outcomes in non-surgically managed AAD patients with hypertension. Large numbers of investigations are required to further explore this relationship.

Keywords: aortic dissection, gender-differences, hypertension, short-term outcomes

\section{Introduction}

Hypertension is considered as one of the most critical risk factors for AAD development, ${ }^{1,2}$ however, no studies have evaluated the relationship between gender difference and short-term outcomes in non-surgically managed AAD patients comorbidity of hypertension. Previous studies showed that about 75 million adults comorbidity with hypertension in the United States, the incidence of AAD is 2.9 to 3.5 per 100,000 person-years. ${ }^{3-6}$ AAD needs a quick diagnosis and appropriate treatment to prevent the fatal complications of AAD. Overall mortality for AAD in the first IRAD publication was about $27.4 \%{ }^{7,8}$ Given the higher mortality rate of AAD, it seems very important to 
identify the characteristics that may predict the prognosis of AAD patients with hypertension.

There are sexer differences in presentation and risk of other cardiovascular health conditions. ${ }^{9,10}$ To date, some studies focused on sex-related differences in short-term or long-term survival in AAD after surgery. ${ }^{1,11-16}$ However, the genderrelated outcome in non-surgically managed with AAD is less well elucidated. Therefore, our objective was to explore the relationship between different gender and short-term outcomes in non-surgically managed AAD of hypertension.

\section{Patients and Methods \\ Study Design and Samples}

This is a retrospective cohort study. Consecutive AAD patients undergoing Computed tomography angiography scanning within 14 days of onset between January 2014 to December 2018 at the Second Xiangya Hospital of Central South University, Changsha, China were eligible to participate in the study. The diagnosis of AAD is based on the 2014 European Society of Cardiology (ESC) guidelines. ${ }^{10}$ Exclusion criteria included patients under 18 years of age, patients with aortic blunt traumatic injury, pregnant patients, patients with a history of AAD surgery, patients with prior history of malignant tumors, non-hypertension patients, patients with surgery management, and patients who visited the hospital after symptoms onset than 14 days. This study was approved by the hospital institutional review board, whereas as a retrospective observational study, informed consent was waived.

\section{Variables Included for Analysis}

The medical information of AAD patients was recorded to collect data on medical history, gender, age, laboratory tests, clinical presentation, imaging studies during admission, and management. All variables were compared between the different gender groups. The diagnosis of hypertension was determined based on the participants' medical records: the doctor's diagnosis or the use of antihypertensive drugs. The Stanford type was determined by imaging. The missing data is replaced by estimates, which were combined using the Rubin rule.

\section{Statistical Methods}

Statistical analyses were performed using the $\mathrm{R}$ version 4.0.3. Continuous variables were shown as median (interquartile range; IQR) or mean (standard deviation; $\mathrm{SD})$. Categorical variables were shown as frequencies. Continuous variables were compared using $t$-test or Mann-Whitney $U$-test. Comparisons between groups for categorical variables were performed using Chi-square analysis or Fisher's exact test. Statistical significance was set at $\alpha=0.05$. We performed the distribution of AAD and in-hospital mortality in different gender of age groups. Some variables associated with in-hospital mortality in different gender were identified for adjustments. Furthermore, three models were used to analyze the association of gender differences and in-hospital mortality in $\mathrm{AAD}$ in the adjusted risks.

\section{Results}

\section{Baseline Characteristics}

A total of 288 patients were included in this study, of whom 238 were male $(82.63 \%)$ and 50 were females $(17.36 \%)$ (Figure 1). The differences in baseline characteristics between women and men are shown in Table 1. Women more likely to be combined with diabetes than men, while men had a higher rate of smoking and drinking $(\mathrm{p}<0.001)$. Age, systolic blood pressure, diastolic blood pressure, heart rate, stroke, atherosclerosis, Marfan syndrome, and chronic kidney were similar between the two groups. Compared with female patients on admission, male patients had more typical symptoms (chest pain, abdominal pain and back pain) and less atypical symptoms (syncope), but there was no significant difference. The typical onset symptoms of women were less than men, such as chest pain, abdominal pain, and back pain, female patients were more likely to present as syncope and other symptoms to hospital. Female patients between the ages of 45 and 68 have a higher incidence of AAD than men (Figure 2A), and this phenomenon is more obvious in male and female in-hospital mortality (Figure 2B).

\section{Laboratory Testing and Imaging Characteristics}

Table 2 illustrates that men have a high level of uric acid and creatinine compared with women. In terms of anatomy, the aortic regurgitation, abdominal vessel, and arch vessel involvement were not different between women and men. Similar, the incidence of cardiac tamponade and pleural effusion were not different between the two 


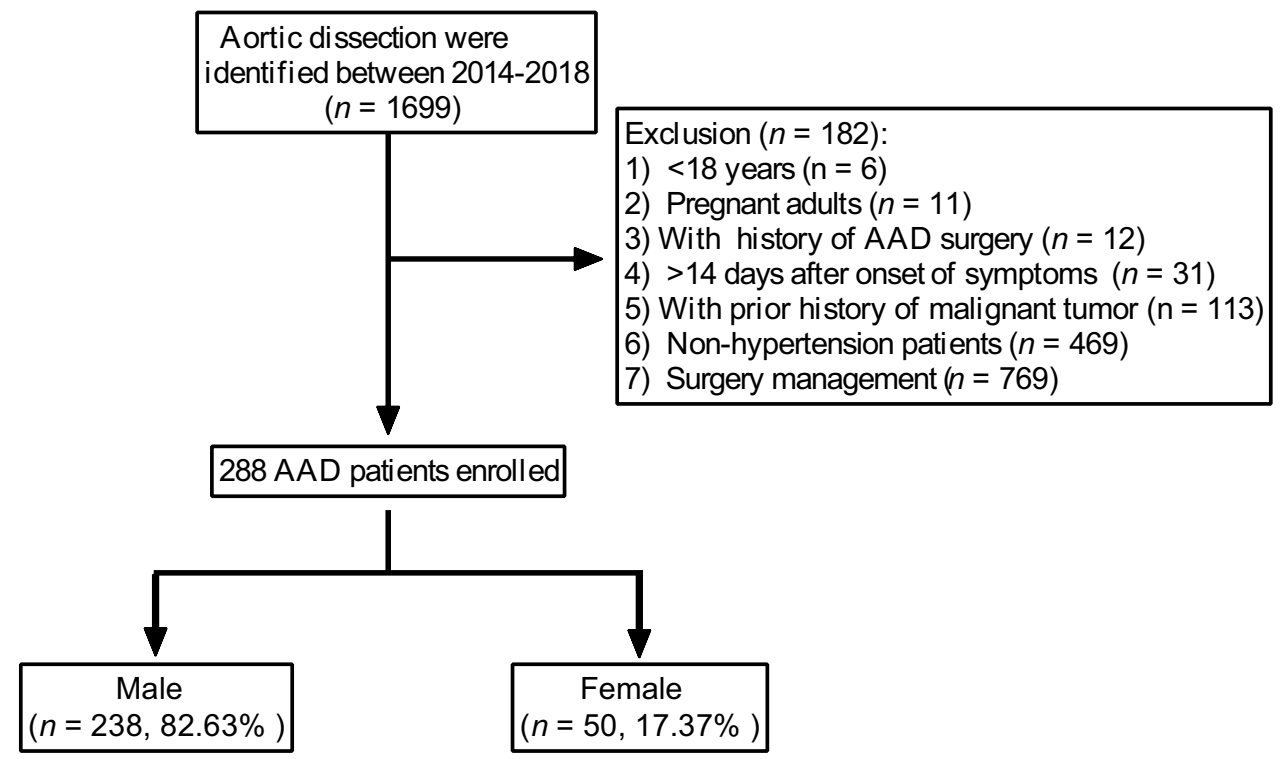

Figure I Flowchart of participant enrollment. Abbreviation: AAD, acute aortic dissection.

groups. No significant gender difference was found in short-term outcomes between acute Type A and type $\mathrm{B}$ aortic dissections, which was consistent with prior research in Chinese AAD patients with medical treatment. ${ }^{14}$

\section{Treatment and Primary Outcomes}

Table 3 shows the medication used to control hypertension and short-term outcomes of two groups of patients. Most of both group AAD patients were used $\beta$-blockers $(72.77 \%$ vs $73.33 \%, \mathrm{P}=0.938$ ), and other medicines used were similar between the two groups. In-hospital mortality was higher in women than men ( $74 \%$ vs $56.3 \%, \mathrm{P}=0.021$, Figure 3$)$.

\section{Kaplan-Meier Analysis for Short-Term Survival in Different Gender}

Follow-up data were available for 288 patients. KaplanMeier analysis showed that the cumulative survival rate

Table I Baseline Characteristics

\begin{tabular}{|c|c|c|c|c|}
\hline Variables & Overall (\%) $n=288$ & Male (\%) $n=238$ & Female (\%) $n=50$ & $P$-value \\
\hline Age, year & $55.72 \pm 12.13$ & $55.30 \pm 12.44$ & $57.74 \pm 10.39$ & 0.196 \\
\hline Systolic blood pressure, $\mathrm{mmHg}$ & $145.85 \pm 31.68$ & $145.40 \pm 30.97$ & $|47.94 \pm 35.1|$ & 0.608 \\
\hline Diastolic blood pressure, $\mathrm{mmHg}$ & $81.75 \pm 19.41$ & $81.63 \pm 19.36$ & $82.32 \pm 19.80$ & 0.819 \\
\hline Heart rate, beats/min & $83.54 \pm 15.70$ & $83.42 \pm 16.21$ & $84.16 \pm 13.04$ & 0.776 \\
\hline Drinking & 38 (13.19) & $36(15.13)$ & $2(4.00 \%)$ & 0.035 \\
\hline Smoking & $91(31.60)$ & $87(36.55)$ & $4(8.00)$ & $<0.001$ \\
\hline Diabetes & $14(4.86)$ & $7(2.94)$ & $7(14.00)$ & $<0.001$ \\
\hline Stroke & $20(6.94)$ & $15(6.30)$ & $5(10.00)$ & 0.350 \\
\hline Atherosclerosis & $40(13.89)$ & $34(14.29)$ & $6(12.00)$ & 0.671 \\
\hline Marfan syndrome & $4(1.39)$ & $3(1.26)$ & I (2.00) & 0.685 \\
\hline Chronic kidney & $8(2.78)$ & $7(2.94)$ & I $(2.00)$ & 0.713 \\
\hline Symptoms & & & & 0.090 \\
\hline Chest pain & $201(69.79)$ & I $70(7 \mid .43)$ & $31(62.00)$ & \\
\hline Abdominal pain & $25(8.68)$ & $22(9.24)$ & $3(6.00)$ & \\
\hline Back pain & $17(5.90)$ & $15(6.30)$ & $2(4.00)$ & \\
\hline Syncope & $5(1.74)$ & $4(1.68)$ & I $(2.00)$ & \\
\hline Other & $40(\mid 3.89)$ & $27(11.34)$ & $13(26.00)$ & \\
\hline
\end{tabular}



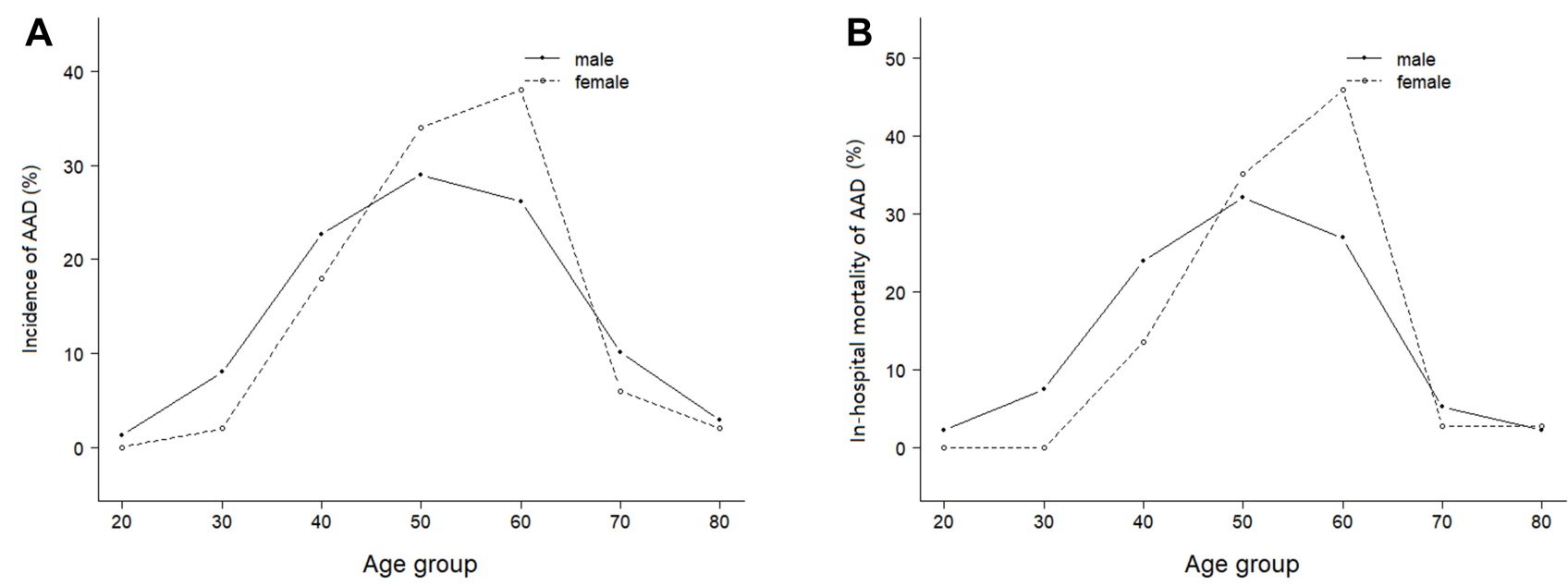

Figure 2 The incidence of AAD and in-hospital mortality in the different age groups. (A) The incidence of AAD with different gender in different age groups. (B) The incidence of in-hospital mortality with different gender in the different age groups.

Abbreviation: AAD, acute aortic dissection.

of female patients in hospital was significantly reduced ( $\log$-rank $\chi 2=8.133, \mathrm{P}=0.004$, Figure 4).

\section{Association Between Gender and Short-Term Outcomes in Non-Surgically Managed AAD Patients with Hypertension}

Multivariable-adjusted effect size and 95\% confidence interval (CI) of short-term outcomes stratified by gender are shown in Table 3. In the full model (model 3), after adjusting for confounding variables, female sex was indicated as an independent risk factor of in-hospital mortality in non-surgically managed AAD patients with hypertension $(\mathrm{OR}=3.60,95 \% \mathrm{CI}=1.41$ to $9.60, \mathrm{P}=0.007$, Figure 5).

\section{Discussion}

In the present study looking at the association of gender with short-term mortality in Chinese AAD patients with hypertension, we found that females (OR, 3.60 in model 3) were significantly associated with short-term outcomes, after adjusting for risk factors. An observational study demonstrated that the mean age of acute aortic dissection was 61 years, ranging from 48 to 67 , and $50 \%$ to $81 \%$ were male. ${ }^{17}$ This research adds clarity to the current literature, which has been partly inconsistent in terms of gender-difference after surgery. ${ }^{11,15}$ Recent studies indicated that gender difference was associated with the outcomes following thoracic aortic surgery ${ }^{11}$ and aortic root replacement. ${ }^{15}$ However, multiple medical centers study indicates that gender did not significantly affect the shortterm clinical outcomes and actuarial survival following repair of type A AAD. ${ }^{12}$ An observational study of 9855 type B AAD patients indicated that compared with men, women were more likely to experience endovascular and sustain a perioperative cardiac event with open surgery. ${ }^{13}$ In Chinese with $\mathrm{AAD}$, gender is also not independently related to long-term clinical outcomes. ${ }^{14}$ Our study may have a better assessment of gender-related short-term outcomes disparity.

In the study from the International Registry of Acute Aortic Dissection (IRAD), women present with higher inhospital mortality. ${ }^{4}$ Women accounted for a smaller cohort in their study, and the types of comorbidities are not substantially different from men, although similar to our research, women tend to be older $(\mathrm{P}<0.01)$. Additionally, Chung et $\mathrm{al}^{11}$ also found female patients were older with larger indexed aortic sizes and have worse outcomes in AAD patients followed by thoracic aortic surgery. Females tended to be older and generally more chronically ill, with higher rates of diabetes. ${ }^{13}$ Though there is no significant statistics difference of age in different genders, females were older and more likely to present with atypical pain than males in our study. Differences in inhabit, phenotype, diet, and customs between Western and Chinese populations might also affect treatment outcomes. ${ }^{14}$ The current study indicates similar results that females were more likely to experience a worse outcome as well.

The potential reasons may be explained as follows. First, women presented later in the course of the disease, with more coma/altered mental status, hypotension, and 
Table 2 Laboratory Testing and Imaging Characteristics

\begin{tabular}{|c|c|c|c|c|}
\hline Variables & Overall (\%) n=288 & Male (\%) n=238 & Female (\%) $n=50$ & $P$-value \\
\hline White blood cell, $\times 10^{9}$ & $11.36 \pm 4.54$ & $11.48 \pm 4.52$ & $10.82 \pm 4.64$ & 0.353 \\
\hline Platelet, $\times 10^{9}$ & $182.03 \pm 80.85$ & $182.02 \pm 75.47$ & $182.08 \pm 104.06$ & 0.996 \\
\hline Uric acid, umol/L, (IQR) & $369.9(276.6,447.35)$ & $373.9(280.12,465.6)$ & $318.3(224.2,419)$ & 0.009 \\
\hline Creatinine, umol/L, (IQR) & $97.9(74.6,148.9)$ & $100.5(77.9,152.6)$ & $79.5(62.4,137.6)$ & 0.007 \\
\hline Prothrombin time, $s$ & $14.29 \pm 2.93$ & $|4.22 \pm 2.3|$ & $14.62 \pm 5.02$ & $0.41 \mathrm{I}$ \\
\hline Activated partial thromboplastin time, $s$ & $39.80 \pm 10.13$ & $40.10 \pm 10.17$ & $38.26 \pm 9.86$ & 0.277 \\
\hline D-dimer, (IQR) & $4.86(2.18,15.10)$ & $4.88(2.26,14.48)$ & $4.17(1.98,17.60)$ & 0.688 \\
\hline Left ventricular ejection fraction, $\%$ & & & & 0.260 \\
\hline $20-40$ & $2(1.23)$ & $2(1.46)$ & $0(0.00)$ & \\
\hline $4 I-60$ & $22(13.50)$ & $16(11.68)$ & $6(23.08)$ & \\
\hline$>60$ & I 39 (85.28) & $119(86.86)$ & $20(76.92)$ & \\
\hline Aortic regurgitation & & & & 0.089 \\
\hline Mild & $6 \mathrm{I}(59.80)$ & $54(62.79)$ & $7(43.75)$ & \\
\hline Moderate & $17(16.67)$ & II (I2.79) & $6(37.50)$ & \\
\hline Severe & $4(3.92)$ & $3(3.49)$ & I (6.25) & \\
\hline Stanford type & & & & 0.839 \\
\hline Type A & $142(49.31)$ & II 8 (49.58) & $24(48.00)$ & \\
\hline Type B & $146(50.69)$ & $120(50.42)$ & $26(52.00)$ & \\
\hline Abdominal vessel involvement & $74(25.69)$ & $60(25.21)$ & $14(28.00)$ & 0.681 \\
\hline Arch vessel involvement & $51(45.95)$ & $44(46.32)$ & $7(43.75)$ & 0.849 \\
\hline Cardiac tamponade & $35(28.23)$ & $26(25.00)$ & $9(45.00)$ & 0.069 \\
\hline Pleural effusion & $64(49.61)$ & $52(46.85)$ & $12(66.67)$ & 0.119 \\
\hline
\end{tabular}

Abbreviation: IQR, interquartile range.

tamponade. ${ }^{4}$ Second, as a previous study illustrated that the underlying pathology may differ between women and men. Women presented with larger indexed aortic sizes. ${ }^{11}$ Women exhibit increased extracellular matrix decomposition by increasing MMP-2 and MMP-9 expression and decreasing inhibitory TIMP-1 and TIMP-2 expression, leading to greater aortic stiffness and faster aortic growth rate. ${ }^{11,18}$ Third, circadian rhythm or weekend admission was related to spontaneous acute dissecting aneurysm rupture or aortic aneurysm rupture in female patients. Studies conducted in Italy either on one of the most important regions (Emilia-Romagna) and the data from the Ministry of Health of Italy as a whole showed that admission on weekends and female gender were independent risk factors for increased in-hospital mortality. ${ }^{19}$ Circadian and seasonal patterns of dissection have been shown also for genetic aneurysms, although with differences by gender. ${ }^{20}$ Moreover, the circadian cycle of spontaneous AAD or rupture of the aortic aneurysm has been proven, which is characterized by a primary peak in the morning and a secondary peak in the evening. ${ }^{21}$ Blood pressure is a crucial triggering factor, and the circadian rhythm of blood pressure is strikingly similar to that found in acute events, since the peaks of blood pressure during the waking hours may be the key to determining the timing of acute dissection and rupture on the weakened aortic

Table 3 Treatment and Primary Outcomes

\begin{tabular}{|l|l|l|l|l|}
\hline Variables & Overall (\%) $\mathbf{n = 2 8 8}$ & Male (\%) $\mathbf{n = 2 3 8}$ & Female (\%) $\mathbf{n = 5 0}$ & P-value \\
\hline Nitrate drugs & $159(55.21)$ & $135(60.27)$ & $24(53.33)$ & 0.767 \\
Angiotensin-converting enzyme inhibitor & $21(7.8 I)$ & $17(7.59)$ & $4(8.89)$ & 0.767 \\
Angiotensin receptor blocker & $86(31.97)$ & $77(34.38)$ & $9(20.00)$ & 0.059 \\
$\beta$-blockers & $196(72.86)$ & $163(72.77)$ & $33(73.33)$ & 0.938 \\
Calcium channel blockers & $151(56.13)$ & $129(57.59)$ & $22(48.89)$ & 0.283 \\
In-hospital mortality & $171(59.38)$ & $134(56.30)$ & $37(74.00)$ & 0.021 \\
\hline
\end{tabular}




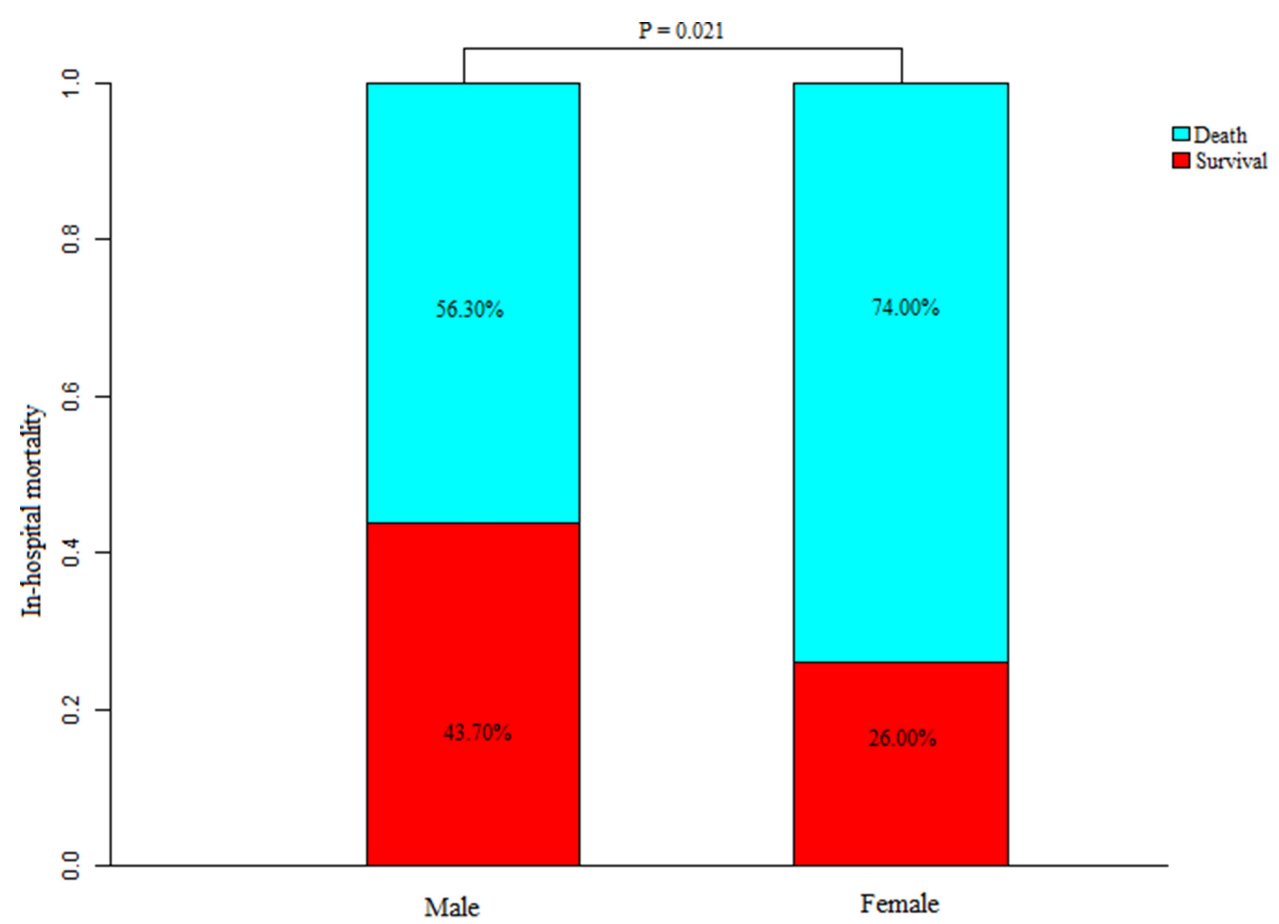

Figure 3 Comparison of in-hospital mortality stratified by the different gender.
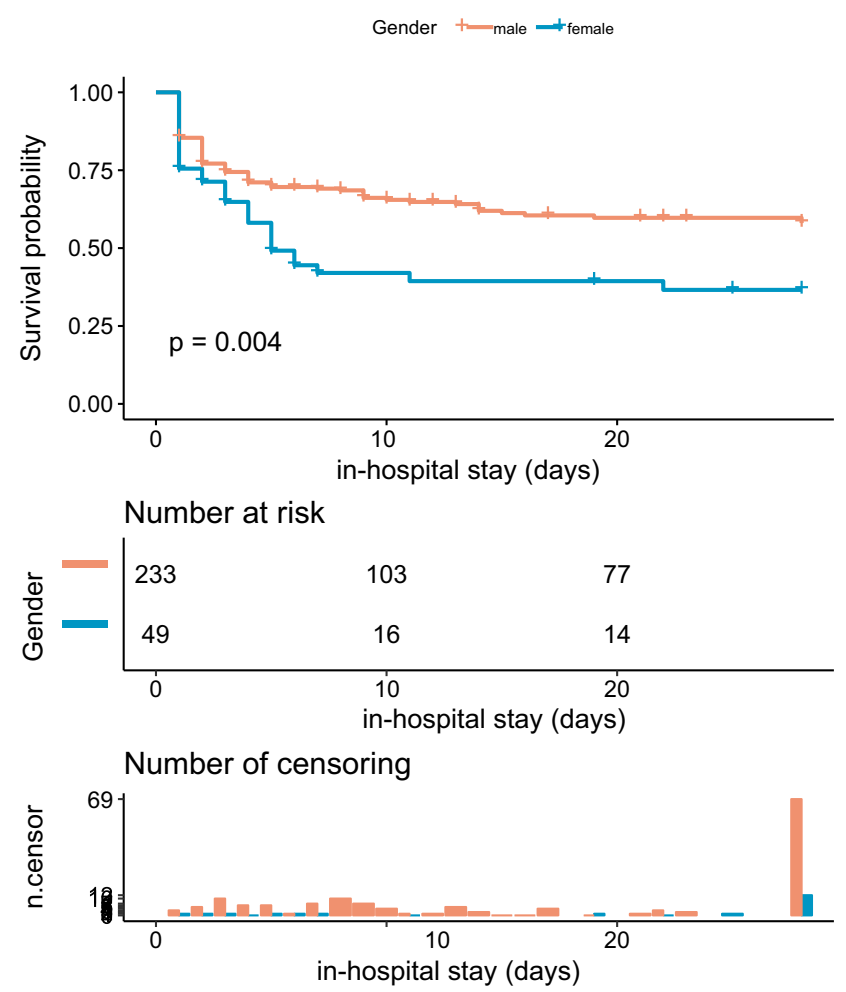

Figure 4 Kaplan-Meier curves for in-hospital survival according to different gender in non-surgically managed acute aortic dissection patients with hypertension.

wall. ${ }^{20}$ Women are associated with higher mortality, not only in the setting of AAD after surgery but also in nonsurgically managed AAD.

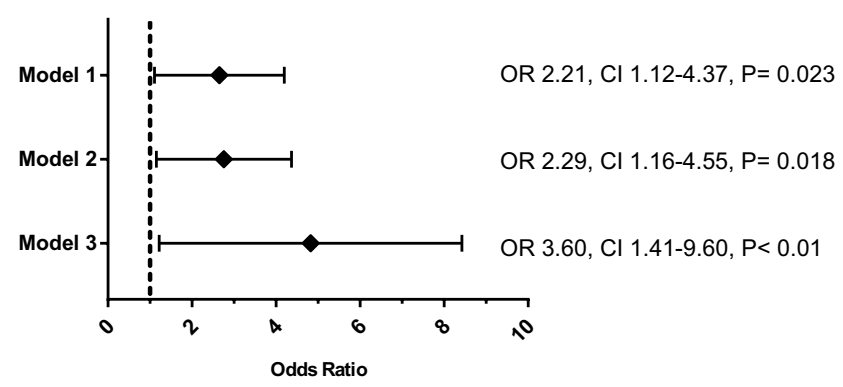

Figure 5 The effect of female sex on outcomes following multivariable analysis. Model I: adjust for none. Model 2: adjust for: age, SBP, DBP. Model 3: age, SBP, DBP, stroke, diabetes, CKD, CHD, drinking, smoking, symptom, type.

Abbreviations: SBP, systolic blood pressure; DBP, diastolic blood pressure; CKD, chronic kidney disease; CHD, coronary heart disease.

However, the study also has some limitations as following, first, due to a retrospective cohort study, which may have some biases on the populations, though our hospital is one of the major-center for AAD in China. Therefore, further researches are needed to examine the relationship between genderdifference and short-term outcomes. Second, due to the limited samples, we did not analyze based on the different types of AAD. But after adjusting the confounders, including age, SBP, DBP, stroke, diabetes, CKD, CHD, drinking, smoking, symptom, Stanford type, the results suggested females are still an independent risk factor for in-hospital mortality in non- 
surgically AAD patients with hypertension. Finally, some large-scale randomized controlled trial studies are needed to be further examined.

Despite these shortcomings, this study is the first study to investigate the association of gender-difference with shortterm outcomes in non-surgically AAD with hypertension. Even for the most experienced clinicians, AAD diagnosis is a daunting task. Given the potentially catastrophic results of delayed detection, the aorta computed tomography angiography (CTA) examination should be performed as soon as possible when female patients suspect dissection. Furthermore, clinicians should alert non-surgically managing females AAD of in-hospital mortality. In the future, further well-controlled studies or multi-centers studies would be helpful to understand better the association of gender-difference and short-term outcomes in non-surgically AAD with hypertension.

\section{Conclusion}

Female patients were more likely to have worse outcomes in non-surgically managed acute aortic dissection patients with hypertension. Large numbers of investigations are required to better explore the discoveries and long-term outcomes.

\section{Data Sharing Statement}

All data generated or analyzed during this study are included in this published article.

\section{Ethics Approval and Consent to Participate}

This study was approved by the hospital institutional review board of the Second Xiangya Hospital. The retrospective data were collected and reviewed in compliance with the ethical standards set out by the Ethics Committee of the institution and with the Declaration of Helsinki. For this retrospective study, data were anonymous and compiled from the hospital electronic medical record system, informed consent was waived. It was also assured that all data were used only for research purposes.

\section{Author Contributions}

All authors contributed to data analysis, drafting or revising the article, have agreed on the journal to which the article will be submitted, gave final approval of the version to be published, and agree to be accountable for all aspects of the work.

\section{Funding}

This work was supported by the Fundamental Research Funds for the Central Universities of Central South University (No.2020zzts291); the Key Research and Development Program of Hunan Province (No.2019SK2022; No.2020SK3004); the Fundamental Research Funds for the Central Universities of Central South University; the Natural Science Foundation of Hunan Province, China (No.2019JJ40451); and the National Natural Science Foundation of China (No.81100221).

\section{Disclosure}

All authors declared no conflicts of interest.

\section{References}

1. Dong N, Piao H, Li B, Xu J, Wei S, Liu K. Poor management of hypertension is an important precipitating factor for the development of acute aortic dissection. J Clin Hypertens. 2019;21:804-812. doi:10.1111/jch.13556

2. Koracevic G, Lovic D, Zdravkovic M, Stojanovic M. Long-lasting, resistant hypertension should be a part of the aortic dissection risk score. Hypertens Res. 2019;42:1836-1838. doi:10.1038/s41440-0190305-8

3. Shirali AS, Bischoff MS, Lin HM, et al. Predicting the risk for acute type $\mathrm{B}$ aortic dissection in hypertensive patients using anatomic variables. JACC Cardiovasc Imaging. 2013;6:349-357. doi:10.1016/ j.jcmg.2012.07.018

4. Nienaber CA, Fattori R, Mehta RH, et al. Gender-related differences in acute aortic dissection. Circulation. 2004;109:3014-3021. doi:10.1161/01.CIR.0000130644.78677.2C

5. Zhou Y, Yang G, He H, Pan X, Peng W, Chai X. Association between admission time and in-hospital mortality in acute aortic dissection patients: a retrospective cohort study. Heart Lung. 2020;49 (5):651-659. doi:10.1016/j.hrtlng.2020.04.005

6. Hagan PG, Nienaber CA, Isselbacher EM, et al. The International Registry of Acute Aortic Dissection (IRAD): new insights into an old disease. JAMA. 2000;283:897-903. doi:10.1001/jama.283.7.897

7. Evangelista A, Isselbacher EM, Bossone E, et al. Insights From the International Registry of Acute Aortic Dissection: a 20-year experience of collaborative clinical research. Circulation. 2018;137:1846-1860. doi:10.1161/CIRCULATIONAHA.117.031264

8. Pape LA, Awais M, Woznicki EM, et al. Presentation, diagnosis, and outcomes of Acute Aortic Dissection: 17-year trends from the International Registry of Acute Aortic Dissection. $\mathrm{J} \mathrm{Am}$ Coll Cardiol. 2015;66:350-358. doi:10.1016/j.jacc.2015.05.029

9. Ostadal B, Ostadal P. Sex-based differences in cardiac ischaemic injury and protection: therapeutic implications. $\mathrm{Br} J$ Pharmacol. 2014;171:541-554. doi:10.1111/bph.12270

10. Regitz-Zagrosek V, Oertelt-Prigione S, Prescott E, et al.; Group EUCCS. Gender in cardiovascular diseases: impact on clinical manifestations, management, and outcomes. Eur Heart J. 2016;37:24-34.

11. Chung J, Stevens LM, Ouzounian M, et al. Sex-related differences in patients undergoing thoracic aortic surgery. Circulation. 2019;139:1177-1184. doi:10.1161/CIRCULATIONAHA.118.035805

12. Conway BD, Stamou SC, Kouchoukos NT, Lobdell KW, Hagberg RC. Effects of gender on outcomes and survival following repair of acute Type A Aortic Dissection. Int $J$ Angiol. 2015;24:93-98. doi:10.1055/s-0034-1396341 
13. Liang NL, Genovese EA, Al-Khoury GE, Hager ES, Makaroun MS, Singh MJ. Effects of gender differences on short-term outcomes in patients with Type B Aortic Dissection. Ann Vasc Surg. 2017;38:78-83. doi:10.1016/j.avsg.2016.06.006

14. Liu YJ, Wang XZ, Wang Y, et al. Correlation between sex and prognosis of Acute Aortic Dissection in the Chinese population. Chin Med J. 2018;131:1430-1435. doi:10.4103/0366-6999.233943

15. McMullen H, Yamabe T, Zhao Y, et al. Sex-related difference in outcomes after aortic root replacement. $J$ Card Surg. 2020;35:1010-1020. doi:10.1111/jocs.14523

16. Rylski B, Georgieva N, Beyersdorf F, et al. Gender-related differences in patients with acute aortic dissection type A. $J$ Thorac Cardiovasc Surg. 2019. doi:10.1016/j.jtcvs.2019.11.039

17. Mussa FF, Horton JD, Moridzadeh R, Nicholson J, Trimarchi S, Eagle KA. Acute Aortic Dissection and intramural hematoma: a systematic review. JAMA. 2016;316:754-763. doi:10.1001/ jama.2016.10026
18. Cheung K, Boodhwani M, Chan KL, Beauchesne L, Dick A, Coutinho T. Thoracic Aortic aneurysm growth: role of sex and aneurysm etiology. $J$ Am Heart Assoc. 2017;6. doi:10.1161/ JAHA.116.003792

19. Gallerani M, Volpato S, Boari B, et al. Outcomes of weekend versus weekday admission for acute aortic dissection or rupture: a retrospective study on the Italian National Hospital Database. Int J Cardiol. 2013;168:3117-3119. doi:10.1016/j.ijcard.2013.04.065

20. Manfredini R, Boari B, Gallerani M, et al. Chronobiology of rupture and dissection of aortic aneurysms. J Vasc Surg. 2004;40:382-388. doi:10.1016/j.jvs.2004.04.019

21. Gallerani M, Portaluppi F, Grandi E, Manfredini R. Circadian rhythmicity in the occurrence of spontaneous acute dissection and rupture of thoracic aorta. J Thorac Cardiovasc Surg. 1997;113:603-604. doi:10.1016/S0022-5223(97)70376-3
Risk Management and Healthcare Policy

\section{Publish your work in this journal}

Risk Management and Healthcare Policy is an international, peerreviewed, open access journal focusing on all aspects of public health, policy, and preventative measures to promote good health and improve morbidity and mortality in the population. The journal welcomes submitted papers covering original research, basic science, clinical \& epidemiological studies, reviews and evaluations,

\section{Dovepress}

guidelines, expert opinion and commentary, case reports and extended reports. The manuscript management system is completely online and includes a very quick and fair peer-review system, which is all easy to use. Visit http://www.dovepress.com/testimonials.php to read real quotes from published authors. 